\title{
A Holistic Approach for the Detection of Media-Adventitia Border in IVUS
}

\author{
Francesco Ciompi ${ }^{1,2}$, Oriol Pujol ${ }^{1,2}$, Carlo Gatta ${ }^{1,2}$, \\ Xavier Carrillo ${ }^{3}$, Josepa Mauri ${ }^{3}$, and Petia Radeva ${ }^{1,2}$ \\ 1 Dep. of Applied Mathematics and Analysis, University of Barcelona, Spain \\ ${ }^{2}$ Computer Vision Center, Campus UAB, Bellaterra, Barcelona, Spain \\ 3 University Hospital "Germans Trias i Pujol", Badalona, Spain \\ fciompi@maia.ub.es
}

\begin{abstract}
In this paper we present a methodology for the automatic detection of media-adventitia border (MAb) in Intravascular Ultrasound. A robust computation of the MAb is achieved through a holistic approach where the position of the MAb with respect to other tissues of the vessel is used. A learned quality measure assures that the resulting MAb is optimal with respect to all other tissues. The mean distance error computed through a set of 140 images is $0.2164( \pm 0.1326) \mathrm{mm}$.
\end{abstract}

\section{Introduction}

Coronary arteries are mainly formed by three layers: intima, media and adventitia. Furthermore, two characteristic membranes can be defined, the Internal Elastic Lamina (IEL) and the External Elastic Lamina (EEL), separating the media from the adventitia layer. In healthy arteries, the shape of the vessel, delineated by the EEL, is approximatively circular, while in unhealthy cases it can become irregular, depending on the plaque amount and positioning. The clinical condition of the artery is commonly characterized by parameters like the Plaque plus media Cross Sectional Area (CSA), the Maximum plaque plus media thickness and the Plaque Burden. All of these measurements require the delineation of the media-adventitia border (MAb).

Intravascular Ultrasound (IVUS) is a catheter-based imaging technique commonly used during percutaneous interventions. It allows to visualize the internal morphology of the vessel, representing a suitable tool for the detection of the MAb. The media layer in IVUS appears as a dark ring surrounding the vessel (Fig1(a)). The thickness of the ring depends on vessel condition, varying from thick region $(800 \mu \mathrm{m})$ in healthy cases to subtle line $(300 \mu \mathrm{m})$ in unhealthy cases, due to the presence of plaque, that pushes the media layer towards the adventitia. Furthermore, the vessel border continuity is often hindered by the guide-wire artifact and by the shadow produced by calcifications. The weak appearance of the media makes the detection of the MAb by IVUS a difficult task.

Several approaches for the automatic MAb detection have been presented so far. In [1], a shape space, together with both calcification and bifurcation detection are used to determine the final border; in [2, anisotropic filtering 


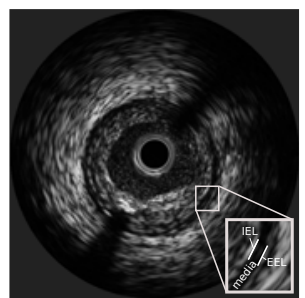

(a)

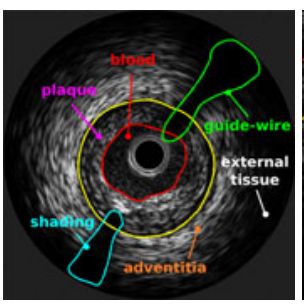

(b)

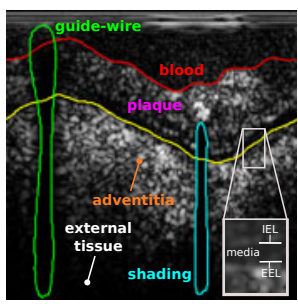

(c)

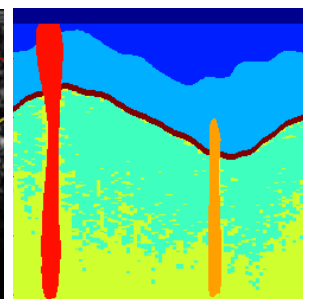

$(d)$

Fig. 1. Example of IVUS image in short-axis view (a); the manually labeled regions are depicted in both cartesian (b) and polar (c) view. In (d) the ground truth labels obtained by processing the borders in (c) are depicted.

operators and classification techniques are combined; in [3], an active contour model is used to regularize the information extracted from the contrast along the edges of the IVUS image; in [4, a Radial Basis Function is applied to the response of a low-pass filtering on the IVUS image; in [5], a snake using gradient vector flow regularizes the map of edges computed by the Canny operator; in [6], a Fast-Marching method based on gray level distributions, assumed as a mixture of Rayleigh pdfs is presented; in [7, the morphological characteristics of the IVUS image are used to initialize an active contour model; finally, in $[8$, deformable models are used, with energy function minimized by a neural network through a simulated annealing scheme.

In this paper we present a robust technique for the automatic detection of the MAb. The methodology is based on two assumptions: (1) only few regions in the image can be recognized, even by an expert physician, as belonging to the media; (2) the MAb can be robustly determined by exploiting the spatial distribution of the information on the morphology of the vessel. For these reasons, the proposed methodology is based on two steps. First, regions belonging to media layer are detected by means of a classification technique. Then, the MAb is detected by considering the relative position of the curve with respect to the surrounding tissues. To the best of our knowledge, this is the first method for the detection of MAb in IVUS based on the relationship between tissues. In particular, not only the local small neighborhood is considered, but all the tissues in the image. As a consequence, the detection of the MAb is the result of the contribution of several informations. This represents the holistic nature of the approach.

\section{Method}

Reflecting the two aforementioned assumptions, the proposed technique consists of two parts. First, the classification of the main morphological regions of the vessel in the IVUS image is performed by means of a multi-class Discriminative Random Fields (DRF) [9]. The usefulness of this step is two-fold. First, by defining the media as one of the classes, an initial approximation of the MAb is obtained (see Fig. 2(b)). Furthermore, the classification of the main regions of the vessel is a necessary information to guide the definition of the MAb where the 


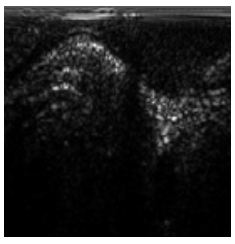

(a)

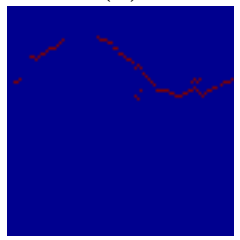

$(f)$

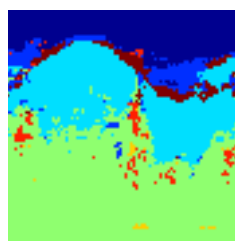

(b)

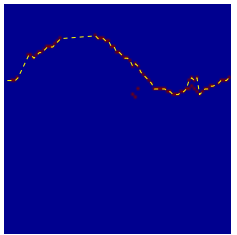

$(g)$

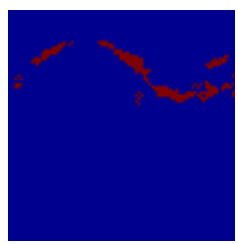

(c)

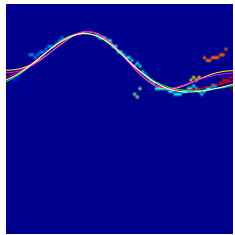

$(h)$

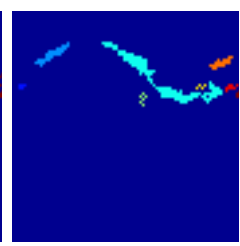

$(d)$

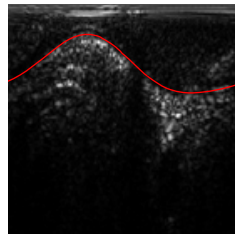

(i)

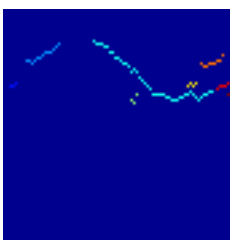

$(e)$

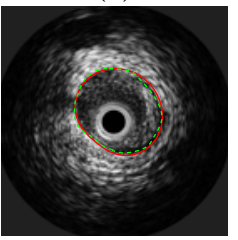

(l)

Fig. 2. Example of the main steps for the MAb detection in polar view $(a-i)$. Each color in (b) represents a class; different colors in (d-e) represent independent isolated media regions. In (l) the detected border (continuous line) and the manual annotation (dotted line) are depicted in the short-axis view.

media appearance is weak or affected by artifacts. The regions labeled as media are often affected by two intrinsic errors: (1) due to the presence of artifacts, the media points are not continuous; (2) media regions can be detected inside the plaque region. Since the adventitia layer is mainly composed by fibrotic tissue, the DRF model learns that the media layer is placed between plaque and fibrosis. For this reason the luminal interface of fibrotic plaque may be labeled as media.

In cases where the MAb position is ambiguous, several guessings can be made. Among them, only one represents the solution to the problem, the optimal curve. Our hypothesis is that the optimal MAb is the one that exhibits the correct relative position with respect to all other tissues. In the second part of the method the points labeled as media are used as initial information with the aim of fitting a continuous and smooth curve. The continuity requirement is first fullfilled by interpolating the discontinuous media regions. Then, the Fourier Series is applied in order to obtain a smooth curve. The relationship between the curve and the tissues is expressed by a quality measure modeled on training examples: the optimal curve is the one producing the highest quality value. The proposed methodology is now explained in details.

\subsection{Multi-class Tissue Classification}

Tissues definition. A set of IVUS frames is manually labelled and used to train a multi-class classifier to discriminate the following classes: blood, plaque, media, adventitia, guide-wire, shadowing and external tissue (see Fig.11for details). The regions corresponding to these classes are derived by the expert manual annotations of lumen border, External Elastic Lamina border (EEL), guide-wire artifact and shadowing effect (due to calcifications). The regions are defined as follows (see Fig. 1 (b)): blood $(L)$ is the area inside the lumen border; adventitia $(F)$ 
is the external area limited by the EEL; plaque $(P)$ is the region comprised between lumen and adventitia; guide-wire $(G)$ /shadow $(S)$ area enclosed in the guide/shadow border; media $(M)$ is defined as a strip of constant thickness $\tau$ internal to the EEL border. In order to assure a sufficient amount of reliable media examples during the training process, the value of $\tau$ has been set as half the thickness of typical unhealthy media layers (around 150-200 $\mu \mathrm{m}$ ). Finally, the class external tissue $(B)$ consists of the set of points labeled as adventitia belonging to a cluster identified by low gray-level value and farther radial position. This procedure can be performed in a similar way in the polar view (see Fig[1(c-d)).

Features. Both textural features and spectral features are extracted from IVUS data, since it has been demonstrated that the combination of both types of information provides an accurate tissue description [10. To this aim, the raw radio frequency data $(\mathrm{RF})$ of each frame is used to form the IVUS image, as detailed in [10]. As in [11, ten textual features are then extracted. Furthermore, the Fast Fourier Transform is applied to the RF data frame, resulting into 32 spectral features, as suggested in 12. For each position of the image a feature vector $\mathbf{x} \in \mathbb{R}^{42}$ is obtained.

Classification. Given the presence of noise and artifacts, the tissues labeling in IVUS is a difficult task. In order to achieve a robust tissue classification, we propose to model the IVUS data as a Discriminative Random Fields (DRF) 9 . In this way the inference process can take into account local information about adjacent tissues. For the design of multi-class DRF, we adopt the recently proposed ECOC-DRF technique [13]. ECOC-DRF is a methodology for the design of both node and edge potential functions for DRF based on the Error-Correcting Output Codes (ECOC) framework [14]. As illustrated in [13, given the number of classes $K$ for the node potential, the distance function for the edge potential is defined over a set of $K^{2}$ classes. In order to reduce the number of dichotomies for the edge potential, while maintaining the properties of the distance function in the ECOC space, we adopt the Sublineal coding technique using binary matrix and Gray coding technique, as suggested in 15. By computing inference over the ECOC-DRF model, the Maximum A-Posteriori probability (MAP) over the seven classes is obtained, and used as input for the MAb detection (Fig. 2(b)).

\subsection{Media-Adventitia Border Detection}

In order to robustly detect the media layer we propose to exploit the information related to the context of the image; we want to take profit of the strong a-priori knowledge about the relative position of the tissues. To this aim, we designed a problem specific quality measure $\Phi$, that takes into account the relative position of the curve with respect to surrounding tissues. The procedure to detect the MAb consists in (1) modeling a set of possible curves and (2) computing a quality measure for each curve. The MAb corresponds to the curve with the highest quality measure.

Firstly, each one of the $M$ isolated regions labeled as media in the MAP (Fig. 2(c)) is considered as an independent element of a set (Fig. 2(d)). Among 
them, only a certain subset delineates the correct vessel border: a region selection process is then required. For this purpose, a number of $D$ (with $D \leq M$ ) regions are considered. Since we are interested in detecting the EEL, for each region, only the points belonging to the farther radial position are used (Fig. 2(e)). Then, the $k$-permutations of $D$ (without repetitions) are computed, where $k=1, \ldots, D$, resulting in $\gamma=D+\sum_{k=2}^{D} \frac{D !}{2(D-k) !}$ combinations. Each combination consists in a set of regions delineating a profile that can be incomplete (Fig. 2(f)). Since the $\mathrm{MAb}$ is a continuous smooth curve, the detected border must fulfill continuity and smoothing constrains. In order to accomplish both requirements, the profile is first approximated by means of a piece-wise linear function (Fig. 2(g)). To assure a smooth curve, by exploiting the periodicity of the function, the Fourier series expansion is computed, and only the first $H \in \mathbb{N}^{+}$harmonics are used. A set of $\gamma$ smooth and continuous borders $b \in B$ is obtained (Fig. 2(h)).

For each curve $b \in B$ in polar coordinates, the normalized amounts of the tissues $T$ positioned above $\left(T_{a}\right)$ and below $\left(T_{b}\right)$ with respect to the curve are computed. Furthermore, let us define $\mathbf{t}(b)^{+}$and $\mathbf{t}(b)^{-}$respectively the vector of correctly and wrongly placed tissues, with respect to the curve $b$. In order to define which are the significative tissues in the MAb detection problem, the following assumptions are done: (1) media points, already used in the border approximation, may be erroneously present in the plaque region; (2) the guide-wire artifact is observable in both region above and below the curve (see Fig. 1(d)). Hence, the classes media and guide-wire do not represent significative markers for curve positioning. For this reason, with reference to the notation in section 2.1. we define $\mathbf{t}(b)^{+}=\left[L_{a}, P_{a}, S_{b}, B_{b}, F_{b}\right]$ and $\mathbf{t}(b)^{-}=\left[L_{b}, P_{b}, S_{a}, B_{a}, F_{a}\right]$. With these assumptions, a quality measure for the curve $b$ is defined as follows:

$$
\Phi(b)=\sum_{i=1}^{m} w_{i}^{+} t_{i}(b)^{+}+\sum_{j=1}^{n}-w_{j}^{-} t_{j}(b)^{-}=\mathbf{w}^{\mathbf{T}} \cdot \mathbf{t}(b)
$$

where $\mathbf{w}=\left[w_{1}^{+}, \ldots, w_{m}^{+}, w_{1}^{-}, \ldots, w_{n}^{-}\right]$is a vector that weights the importance of different contributions to the quality measure. The solution is $\tilde{b}=\operatorname{argmax}(\Phi(b))$.

The estimation of the vector $\mathbf{w}$ is a critical point; its role is two-fold: $(i)$ to assign the proper contribution to each tissue, but also $(i i)$ to take into account of wrong contributions due to errors in the labels. For this purpose, based on training examples, we compute $\mathbf{w}_{\mathbf{o p t}}=\operatorname{argmax} \sum_{v} \mathbf{w}^{T} \cdot \mathbf{t}^{v}\left(b_{G T}\right)$, where $\mathbf{t}^{v}\left(b_{G T}\right)$ is the vector of tissues of the $v^{t h}$ example in a validation dataset and $b_{G T}$ is the ground truth border provided by experts. Given that the optimization problem is a maximization problem of an unbounded function, it is reasonable to constrain the values of the weights as $\|\mathbf{w}\|_{2}^{2}=1$, assuring non trivial solutions. The problem is solved by means of Lagrange multipliers.

\section{Validation}

Experimental Settings. A set of 18 in-vivo pullbacks have been acquired using a Galaxy II IVUS equipment (Boston Scientific) with a catheter Atlantis SR Pro $40 \mathrm{MHz}$ (Boston Scientific). RF data have been sampled with a 12 bit sampling 
Table 1. State-of-the-art methods vs holistic approach (HoliMAb). Details on the number of patients and images used in the validation are provided for all the methods, as well as the ultrasound frequency $(\mathrm{MHz})$ of the used technology. $\mathrm{MDE}=$ mean distance error; $\mathrm{MADE}=$ maximum distance error; $\mathrm{MAE}=$ mean area error; $\mathrm{IOV}=$ inter observer variability.

\begin{tabular}{|l||c|c||c|c|c||c|}
\hline & $\underline{6}$ & $\underline{1}$ & $\underline{7}$ & {$[2$} & HolimAb & IOV \\
\hline Equipment & Jomed & Volcano & Clearview & Clearviews & Galaxy II & -- \\
\hline \# Patients & 9 & 18 & 9 & 11 & 18 & -- \\
\hline \# Images & 540 & 647 & 50 & 5400 & 140 & -- \\
\hline MHz & 20 & 20 & 40 & 40 & 40 & - \\
\hline MDE $(\mathrm{mm})$ & $0.12(0.07)$ & $0.09(0.16)$ & - & $0.2265(0.0688)$ & $0.2164(0.1326)$ & $0.1226(0.1654)$ \\
\hline MADE $\left(\mathrm{mm}^{2}\right)$ & - & $0.98(1.78)$ & $0.07(0.78)$ & $0.5715(0.2296)$ & $0.5162(0.3209)$ & $0.3717(0.3117)$ \\
\hline MAE $\left(\mathrm{mm}^{2}\right)$ & $-0.2(2.1)$ & $0.75(1.60)$ & $0.17(4.58)$ & $8.6032(3.3436)$ & $0.5635(0.4830)$ & $0.3489(0.4405)$ \\
\hline
\end{tabular}

card at a sampling rate of $200 \mathrm{MHz}$. The most representatives and not consecutive challenging frames for each pullback have been selected and manually segmented by three experts, resulting in 140 frames.

Due to computational complexity in performing inference in a graphical model, we construct the lattice by reducing each feature map by a factor $\frac{1}{W_{n}^{2}}$. We assign the median value of each feature contained into non-overlapping blocks of $W_{n} \times W_{n}$ pixels of the image to each node of the graph. Thus, the parameter $W_{n}$ represents a trade-off between computational cost and quality of the MAb estimation. As in [13] the edge feature function is set to $\tilde{\mathbf{x}}=\left|\mathbf{x}_{i}-\mathbf{x}_{j}\right|$. ECOCDRF has been trained according to the Leave-One-Patient-Out (LOPO) scheme [10]: at each fold, one of the patients has been used for testing, and the rest is used for training. For the training process, the manual annotations of the expert exhibiting the minimal mean inter-observer variability are used. The used coding technique is OneVsOne for the node potential and Sublineal coding for the edge potential, requiring 21 and 12 dichotomies, respectively. The margin classifier for the ECOC-DRF has been set as suggested in [13. The used decoding technique is the Attenuated Euclidean Distance for the node potential and the Euclidean Distance for the edge potential; the parameters $\left(\alpha_{N}, \alpha_{E}\right)$ are set as described in [13. The inference is performed by means of Belief Propagation (BP) [16].

Results Three error measures have been computed: (1) Mean Distance Error (MDE), computed by averaging the absolute difference between automatic border and manual annotation per A-line; (2) MAximum Distance Error (MADE), computed by averaging the error on the maximum distance for each frame; (3) Mean Area Error (MAE), computed by averaging the absolute area differences. Parameters tuning, according to the LOPO scheme, provided $D=8$ and $H=2$. In order to determine $D$, the media regions have been ranked according to their length. As expected, the number of Fourier harmonics is small. Since the number of harmonics is inversely proportional to the regularization degree, this result confirms: $(i)$ the hypothesis of assuming a continuous smooth curve and $(i i)$ the shape of the vessel is often ellipsoidal. Figure 3 shows some graphical examples of border detection. Table 1 shows the performance of the proposed method when compared with other state of the art approaches and with the Inter Ob- 


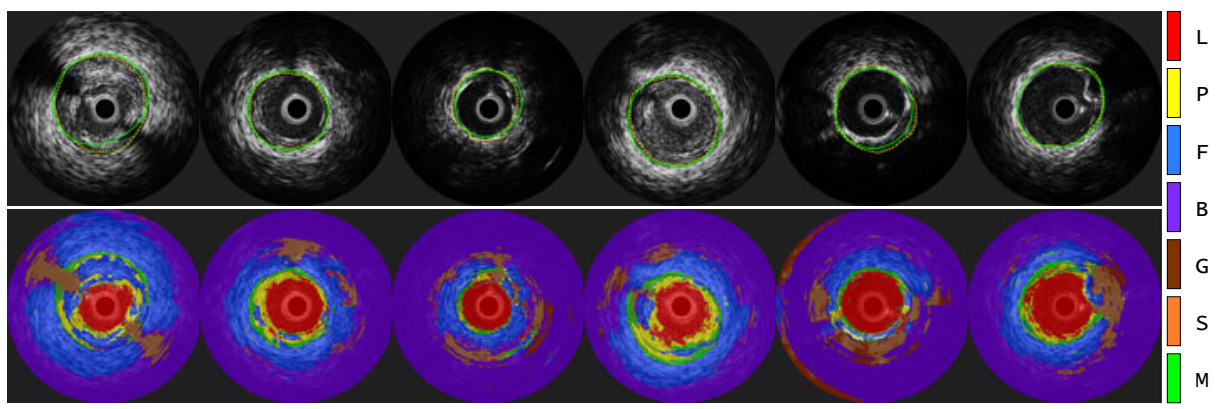

Fig. 3. Examples of automatic MAb detection (above) and corresponding classification maps (below). Continuous line represents the automatic segmentation; dotted line represents the manual annotation; labels names are assigned as in section 2.1

server Variability. The performance of the HoliMAb method is obtained using $W_{n}=3$ : this value experimentally demonstrates to be a reasonable trade-off between quality and computational cost.

\section{Discussion}

The comparison in Table 1 refers to the results provided in the cited paper: the database used in each case is different. In order to perform a fair comparison it is worth to note that some of the methods work on a $20 \mathrm{MHz}$ US signal, while others work on $40 \mathrm{MHz}$. The difference between the two signals is remarkable, since the $20 \mathrm{MHz}$ US exhibits higher tissue penetration power, while losing in local resolution. This fact increases the discrimination of tissues while losing details (but also artifacts) in IVUS data. The differences between the two technologies is evident since the errors obtained with $20 \mathrm{MHz}$ techniques are smaller than IOV. To corroborate this assertion, it is worth to note that in [1] the authors claim that their approach does not work on a $40 \mathrm{MHz}$ technology.

The standard deviation of all the considered error measures in HoliMAb is comparable with the inter-observer variability. This fact demonstrates the robustness of the proposed method that, exploiting the contextual information of the tissues positions, limits the range of error in the detection of the curve. The MDE value is comparable with the approach proposed in [2] that, given the experimental setup, can be considered as an exhaustive clinical study. The value of the MDE error can be controlled by two parameters: (1) the $W_{n}$ value introduces a systematic error due to the change of scale; (2) the number of harmonics $H$ controls the degree of regularization of the curve. The error introduced by the two parameters also represents a limitation of the method. In few cases where a calcification or a dense fibrosis surrounds almost completely the vessel, the method could perform sub-optimally. Finally, it is worth to note that by ranking the obtained values of $\Phi$, several MAb can be proposed to physicians. Alternative solutions can be selected in case the first one is not satisfactory, avoiding the manual delineation of a better MAb. 


\section{Conclusions and Future Work}

A novel methodology for the media-adventitia border detection based on contextanalysis has been presented. The algorithm consists in a constant number of steps, tunable through the parameters $\left(W_{n}, D\right)$, that provide a trade-off between the detection quality and the execution time: for this reason, it can potentially run in real time. The method achieved results comparable with state-of-theart and with inter-observer variability. The whole formulation of the mediaadventitia border detection is based on RF data analysis: the method is then totally independent on the IVUS equipment technology and brand. Nevertheless, given the scarce accessibility to such data, the analysis of the usefulness of RFbased features through features selection is a straightforward step in our research. Furthermore, the applicability of the methodology to $20 \mathrm{MHz}$ IVUS data as well as exploiting spatial information in the vessel morphology by using consecutive frames is part of our future lines. It is worth to note that, though applied to the specific MAb detection problem, the functional in Eq. 1 is of general applicability for the segmentation of each tissue in medical imaging and, more in general, of any object in images where a frontier can be defined.

\section{References}

1. Unal, G., Bucher, S., Carlier, S., Slabaugh, G., Fang, T., Tanaka, K.: Shape-driven segmentation of the arterial wall in intravascular ultrasound images. TITB 12(3), 335-347 (2008)

2. Gil, D., Hernandez, A., Rodriguez, O., Mauri, J., Radeva, P.: Statistical strategy for anisotropic adventitia modelling in ivus. TMI 25, 768-778 (2006)

3. Zhu, H., Liang, Y., Friedman, M.H.: Ivus image segmentation based on contrast. In: SPIE, vol. 4684, pp. 1727-1733 (2002)

4. Papadogiorgaki, M., Mezaris, V., Chatzizisis, Y.S., Giannoglou, G.D., Kompatsiaris, I.: Automated ivus contour detection using intensity features and radial basis function approximation. In: CBMS (2007)

5. Sanz-Requena, R., Moratal, D., Garca-Snchez, D.R., Bod, V., Rieta, J.J., Sanchis, J.M.: Automatic segmentation and $3 \mathrm{~d}$ reconstruction of intravascular ultrasound images for a fast preliminar evaluation of vessel pathologies. CMIG 31(2), 71-80 (2007)

6. Cardinal, M.H.R., Meunier, J., Soulez, G., Maurice, R.L., Therasse, E., Cloutier, G.: Intravascular ultrasound image segmentation: a three-dimensional fastmarching method based on gray level distributions. TMI 25(5), 590-601 (2006)

7. Giannogloua, G.D., Chatzizisisa, Y.S., Koutkiasb, V., Kompatsiarisc, I., Papadogiorgakic, M., Mezarisc, V., Parissic, E., Diamantopoulosd, P., Strintzisc, M.G., Maglaverasb, N., Parcharidisa, G.E., Louridasa, G.E.: A novel active contour model for fully automated segmentation of intravascular ultrasound images: In vivo validation in human coronay arteries. CBM 37, 1292-1302 (2007)

8. Plissiti, M.E., Fotiadis, D.I., Michalis, L.K., Bozios, G.E.: An automated method for lumen and media-adventitia border detection in a sequence of ivus frames. TITB 8(2), 131-141 (2004)

9. Kumar, S., Hebert, M.: Discriminative random fields. IJCV 68(2), 179-201 (2006)

10. Ciompi, F., Pujol, O., Gatta, C., Rodriguez-Leor, O., Mauri-Ferre, J., Radeva, P.: Fusing in-vitro and in-vivo intravascular ultrasound data for plaque characterization. IJCI 26, 763-779 (2010) 
11. Ciompi, F., Pujol, O., Fernandez-Nofrerias, E., Mauri, J., Radeva, P.: Ecoc random fields for lumen segmentation in radial artery ivus sequences. In: Yang, G.-Z., Hawkes, D., Rueckert, D., Noble, A., Taylor, C. (eds.) MICCAI 2009. LNCS, vol. 5762, pp. 869-876. Springer, Heidelberg (2009)

12. Sathyaranayana, S., Carlier, S., Wenguang, L., Thomas, L.: Characterization of atherosclerotic plaque by spectral similarity of radiofrequency intravascular ultrasound signals. EuroIntervention 5, 133-139 (2009)

13. Ciompi, F., Pujol, O., Radeva, P.: A meta-learning approach to conditional random fields using error-correcting output codes. In: IEEE International Conference on Pattern Recognition (ICPR), pp. 710-713 (2010)

14. Dietterich, T.G., Bakiri, G.: Solving multiclass learning problems via errorcorrecting output codes. JAIR 2, 263-286 (1995)

15. Bautista, M.A., Baro, X., Pujol, O., Radeva, P., Vitria, J., Escalera, S.: Compact evolutive design of error-correcting output codes. In: ECML (2010)

16. Yedidia, J.S., Freeman, W.T., Weiss, Y.: Understanding belief propagation and its generalizations. (TR-2001-22), pp. 239-269 (2002) 